\title{
Cone-beam Computed Tomography-guided Stereotactic Liver Punctures: A Phantom Study
}

\author{
Grzegorz Toporek • Daphné Wallach • \\ Stefan Weber · Reto Bale $\cdot$ Gerlig Widmann
}

Received: 7 January 2013/Accepted: 10 March 2013/Published online: 14 May 2013

(C) Springer Science+Business Media New York and the Cardiovascular and Interventional Radiological Society of Europe (CIRSE) 2013

\begin{abstract}
Purpose Images from computed tomography (CT), combined with navigation systems, improve the outcomes of local thermal therapies that are dependent on accurate probe placement. Although the usage of CT is desired, its availability for time-consuming radiological interventions is limited. Alternatively, three-dimensional images from C-arm cone-beam CT (CBCT) can be used. The goal of this study was to evaluate the accuracy of navigated CBCT-guided needle punctures, controlled with CT scans. Methods Five series of five navigated punctures were performed on a nonrigid phantom using a liver specific navigation system and CBCT volumetric dataset for planning and navigation. To mimic targets, five titanium screws were fixed to the phantom. Target positioning accuracy $\left(\mathrm{TPE}_{\mathrm{CBCT}}\right)$ was computed from control CT scans and divided into lateral and longitudinal components. Additionally, CBCT-CT guidance accuracy was deducted by performing CBCT-to-CT image coregistration and measuring $\mathrm{TPE}_{\mathrm{CBCT}-\mathrm{CT}}$ from fused datasets. Image coregistration was evaluated using fiducial registration error $\left(\mathrm{FRE}_{\mathrm{CBCT}-\mathrm{CT}}\right)$ and target registration error $\left(\mathrm{TRE}_{\mathrm{CBCT}-\mathrm{CT}}\right)$.
\end{abstract}

G. Toporek · D. Wallach $(\bowtie) \cdot$ S. Weber

ARTORG Center for Biomedical Engineering Research, University of Bern, Murtenstrasse 50, 3008 Bern, Switzerland

e-mail: daphne.wallach@artorg.unibe.ch

G. Toporek

e-mail: grzegorz.toporek@artorg.unibe.ch

R. Bale · G. Widmann

Section of Microinvasive Therapy, Department of Radiology, Innsbruck Medical University, Anichstrasse 35, 6020 Innsbruck, Austria
Results Positioning accuracies in lateral directions pertaining to $\mathrm{CBCT}\left(\mathrm{TPE}_{\mathrm{CBCT}}=2.1 \pm 1.0 \mathrm{~mm}\right.$ ) were found to be better to those achieved from previous study using CT $\left(\mathrm{TPE}_{\mathrm{CT}}=2.3 \pm 1.3 \mathrm{~mm}\right)$. Image coregistration error was $0.3 \pm 0.1 \mathrm{~mm}$, resulting in an average TRE of $2.1 \pm 0.7 \mathrm{~mm}(N=5$ targets $)$ and average Euclidean ТРЕ $_{\text {Свст-Ст }}$ of $3.1 \pm 1.3 \mathrm{~mm}$.

Conclusions Stereotactic needle punctures might be planned and performed on volumetric CBCT images and controlled with multidetector CT with positioning accuracy higher or similar to those performed using CT scanners.

Keywords Cone-beam computed tomography · Radiological interventions · Ablations

\section{Introduction}

Percutaneous ablation for selected patients with hepatic malignancies is a promising treatment method that might become a less-invasive alternative to surgical resection [1]. However, local therapy procedures, such as percutaneous ablations, are highly dependent on accurate placement of single or multiple needle-like applicators to provide effective clinical outcomes [2].

To enable accurate placement of the ablation probes, the use of image guidance based on ultrasound (US), computed tomography (CT), or magnetic resonance imaging (MRI) is increasingly becoming a standard of interventional radiology practices [3], with each modality having advantages and drawbacks.

Although real-time US imaging is efficient and costeffective, image quality often is obscured by gas bubbles generated during the interstitial thermal ablation and respiratory motion or patient positioning may impair US- 
CT image coregistration accuracy $[4,5]$. MRI is expensive because it requires special MR-compatible equipment, which is not available from most ablation-probe manufactures [6]. On the other hand, thanks to good visualization of hepatocellular carcinoma (HCC) in arterial and portal-venous phase contrast imaging and the best available geometrical properties, CT has become a "gold standard" for minimally invasive percutaneous needle ablations [7-9]. Such procedures are usually performed in a step-by-step approach; this means that several control CT scans need to be acquired before the ablation probe reaches its target. Consequently, the patient safety due to high radiation dose overexposure is sacrificed.

To overcome those limitations, stereotactic instrument guidance systems were developed for minimally invasive, CT-image-based percutaneous interventions [3, 10, 11]. These techniques are based on sensing the positions of both surgical instruments (coaxial needles, thermal therapy applicators) and patient anatomy to codisplay objects from the real clinical situation onto the medical image datasets. To achieve this, a registration (usually rigid) between the intraoperative situation and preoperatively acquired images is performed. Unfortunately, under this assumption, softtissue deformation and patient motion may greatly affect the guidance information provided by navigation system, thus leading to significant inaccuracies depending on the amount of target motion relative to the initial scenario from preoperative imaging. Therefore, the most intuitive solution is to acquire preoperative images shortly before the procedure and perform the intervention directly on the CT table. The major concern with this proposal is that the availability of CT scanners, especially for time-consuming radiological interventions, is limited during the day in most hospital facilities.

Alternatively, C-arm cone-beam computed tomography (CBCT)-imaging technology, available in most modern interventional suites, which incorporates a flat-panel detector (FPD) angiography system to provide either 2D fluoroscopy or 3D volumetric data [12] might be coupled with stereotactic instrument guidance thereby removing the need for intraoperative CT.

Employing C-arm CBCT devices for minimally-invasive percutaneous lesion targeting is not new. However, unlike stereotactic navigation, recent solutions require realtime, 2D fluoroscopy images to be codisplayed with 3D CBCT volumetric data [2, 13-15]. Unfortunately, this approach introduces high irradiation to the medical staff and the patient.

Navigated CBCT-guided stereotactic needle interventions that are independent from online fluoroscopic imaging could overcome problems relating to radiation overexposure. By employing volumetric CBCT image datasets instead of CT datasets, procedures also could become more cost-effective, thereby increasing the popularity of instrument guidance techniques among the interventional radiology community. The positing accuracy pertaining to the use of CBCT for stereotactic guidance is, to our knowledge, yet to be evaluated.

Furthermore, within CBCT images tumors and soft tissue are not always clearly visible, ensuing that CBCT images would need to be fused with conventional diagnostic CT images, possibly increasing inaccuracy in the target definition.

Subsequently, the goal of this study was twofold: (1) to evaluate whether the geometrical accuracy and image quality of CBCT images are sufficient for intraoperative treatment planning and stereotactic percutaneous needle punctures with an optical guidance system, and (2) to compare achieved needle positioning accuracy based on CBCT images to those performed previously on CT datasets [16]. As a secondary endpoint, quantitative information about the achieved fusion accuracy between preoperative CBCT and control CT scans using a fiducial marker-based rigid image coregistration method is reported. Such knowledge might be required if the visibility of lesions and soft tissue on the CBCT scan is not sufficient for intervention planning.

Within this work, the proposed workflow and imaging protocol for stereotactic-guided procedures based on CBCT is presented. Additionally, an accuracy evaluation on a nonrigid phantom, providing comparative results to CTguided procedures is given.

\section{Materials and Methods}

A navigation system dedicated to image-guided open-liver surgical resections and ablations has been adapted to perform CT-guided percutaneous radiological interventions [17]. When the system was combined with the ATLAS aiming device (ATLAS, Elekta AB, Sweden), high needle placement accuracy in the lateral direction has been shown previously [16]. To validate the usability and accuracy of CBCT guidance, in comparison to CT guidance, the aforementioned navigation system was augmented for CBCT volumetric image guidance and a similar positioning accuracy experimental study was designed [16].

\section{Navigation System and Aiming Device}

The navigation system consists of an optical position measurement system (NDI Vicra, Northern Digital, Canada), a touch-based user interface and a set of custom-made marker shields (camera dynamic references) with retroreflective passive markers that can be adapted to a variety of 


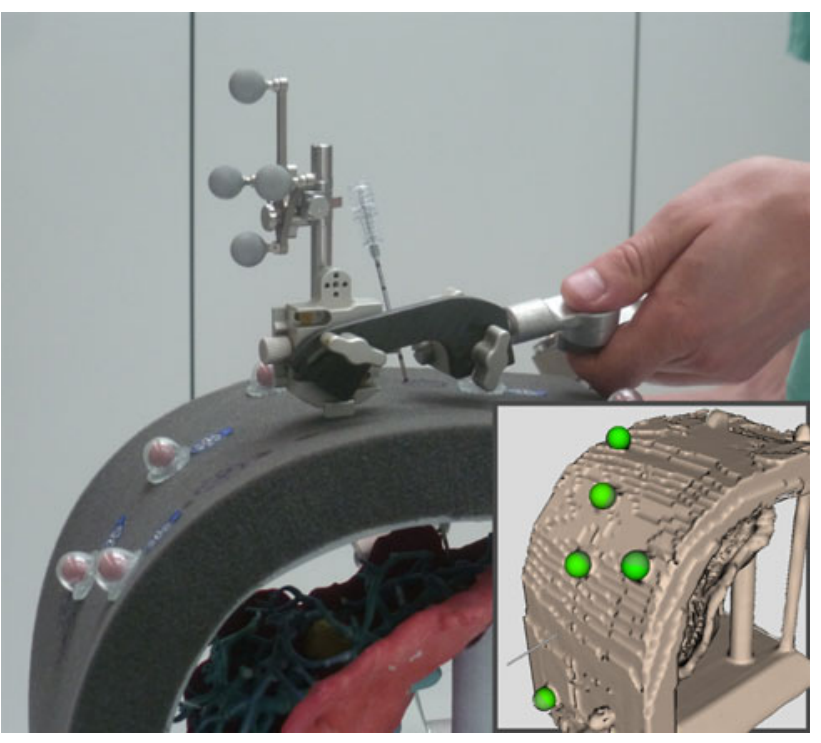

Fig. 1 Navigated adjustment of the ATLAS aiming device over a nonrigid rapid prototyped liver phantom. 3D visualization of used markers configuration (green spheres) is shown in the bottom right corner

tools to enable accurate tracking within the radiological suite. Moreover, at the beginning of the intervention, a combination of single-marker (SM) retroreflective spheres is glued to the patient's skin around the expected needle entry point. The SM sphere positions are automatically detected from the preoperative CBCT image dataset using an optimized three-dimensional Hough transform, described in detail in [18] and rigidly registered to the positions detected by the camera [19]. Real-time patient tracking and respiratory motion control during the interventions is thus achieved.

In order to provide a reliable stabilization during the adjustment and fixation of the final orientation of the needle during the insertion, the ATLAS aiming device is employed [20]. In a standard setup, the aiming device is attached to a carbon plate placed below the patient. To bring the aiming device to the desired entry position, an 8-mm-thick, rigid, medical-grade titanium cylinder, with a rigidly attached marker shield, is placed in the insertion brackets of the aiming device and tracked by the camera (Fig. 1). The alignment of the arm is performed by rotating the holding-brackets around two axes whilst using the visual feedback from the navigation interface. Once the position and angle of the aiming device correspond to the planned trajectory, the axial distance between the cylinder tip and the target is displayed on the screen of the navigation system. This distance is then marked with a biocompatible pen onto the needle. The cylinder is removed from the aiming device and replaced by the needle, which is then inserted up to the indicator line (Fig. 2).

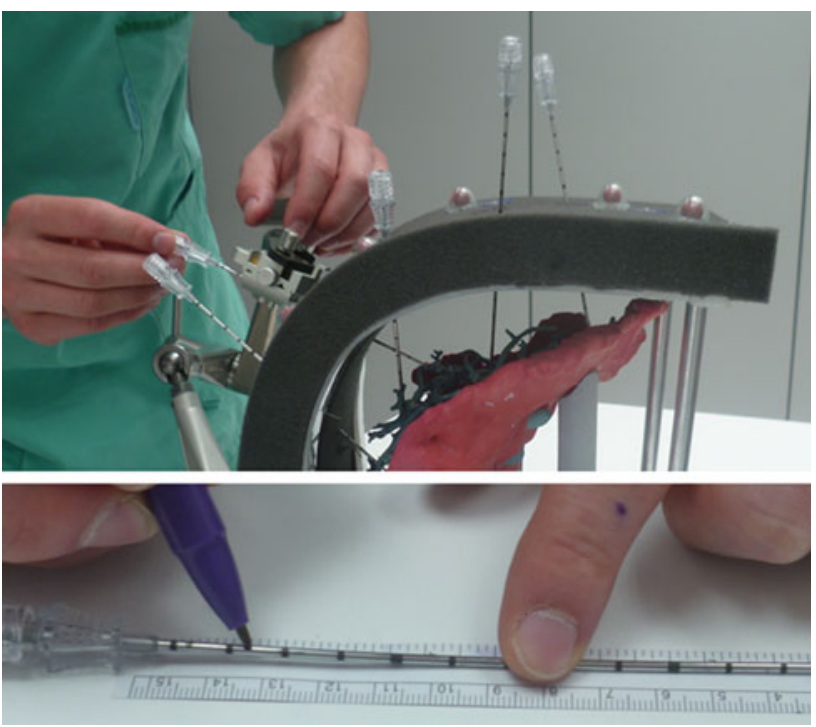

Fig. 2 Insertion of the coaxial needle into the phantom using aiming device. Depth of the needle insertion is controlled passively from the mark placed using a biocompatible pen

\section{Experiment}

In order to validate the usability and accuracy of CBCTguided needle interventions, five series of five needle punctures each were performed on a nonrigid, anthropomorphic liver phantom and evaluated with five control CT scans.

\section{Nonrigid Phantom}

A phantom was produced by rapid prototyping a 3D model of the human liver (segmentation performed by MeVis Distant Services, Bremen, Germany). The model consists of several rigid anatomical structures: vessels, portal and hepatic vein, as well as tumors. Additionally, five $1.5 \times$ 3-mm titanium screws (M-5220.03, Medartis, Switzerland) were fixed on to the tumors of the liver model with the center of the screw heads acting as targets. The liver was placed $\sim 50 \mathrm{~mm}$ beneath deformable plastic foam (Fig. 1) to simulate the patient skin. With the presence of this artificial skin, the phantom mimics real, in vivo situations in that it is essentially impossible to reposition the needle once it is inserted.

Within a clinical setting, a positioning template is used to ensure reproducible placement of the single markers on the patient. The template is placed on the patient's lower thorax region, partially overlapping the abdomen. This template is designed to position the six markers cranially and medially to the prospective insertion area. The average distance between markers is $60 \mathrm{~mm}$, and the area covered by the markers is $\sim 260 \times 150 \mathrm{~mm}$. The template was mainly designed to avoid ambiguities of inter-SM-distances and ensure 
asymmetric placement. For improved target registration accuracy and for decreased interference with the situs, the markers are positioned with a large spread, whilst remaining within the working volume of the optical tracking camera. In this study, the markers were attached to the surface of the phantom according to the clinical template, approximately at 30-40 mm distance from needle entry positions, surrounding the underlying targets.

\section{Imaging Systems and Protocols}

A preoperative, CBCT volumetric dataset used for planning and navigation was acquired using a $\mathrm{C}$-arm angiography flatpanel system (Allura Xper FD20, Philips, The Netherlands), which provides images in quality corresponding to conventional CT images with a maximal field of view (FOV) of $30 \times 38 \mathrm{~cm}$ and a resolution of $0.48 \times 0.48 \times 0.48 \mathrm{~mm}$. The scanning protocol was as follows: abdomen low-dose mode, $119 \mathrm{kVp}, 188 \mathrm{~mA}$ tube current, rotation time $10 \mathrm{~s}, 30$ frames per second. An iterative reconstruction approach, providing higher quality images than those obtained using a standard Feldkamp algorithm, was used. An example of the image quality of the scanned liver phantom compared to standard multidetector computed tomography (MDCT) scanner is depicted in Fig. 3.

Control scans were acquired using a MDCT scanner (Siemens SOMATOM Sensation, Siemens AG, Erlangen, Germany) and used for further error evaluation. The employed acquisition scheme (typical for CT-guided radiological interventions on the liver) was as follows: helical scan, $24 \times 1.2-\mathrm{mm}$ collimation, spatial resolution of $0.65 \times 0.65-\mathrm{mm}$ and $3-\mathrm{mm}$ slice thickness, pitch 1.0, $0.5 \mathrm{~s}$ rotation time, $120 \mathrm{kVp}, 221 \mathrm{~mA}$ tube current.

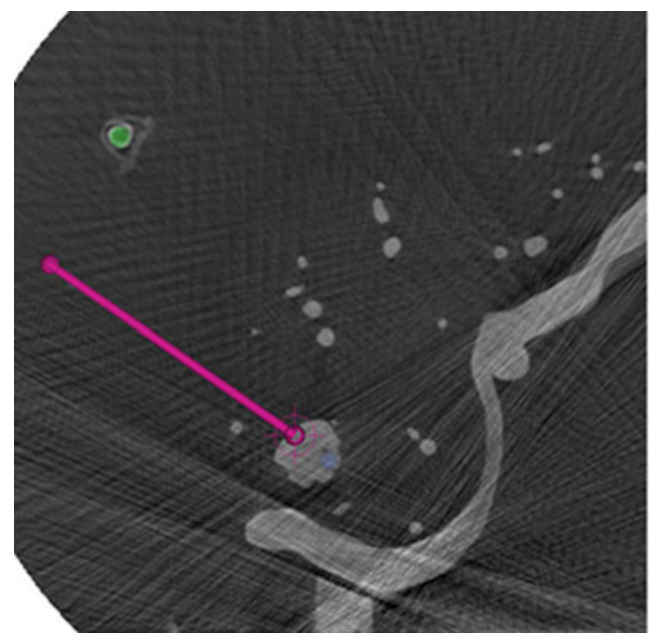

Fig. 3 Screenshot from the application software presenting one CBCT (left) and MDCT (right) slice. CBCT images suffer from streak artifacts and present low signal-to-noise ratio compared to MDCT
Procedure

Preoperative $\mathrm{CBCT}$ of the phantom was acquired and sent via the DICOM network protocol to the navigation system. Five of the six SM were visible on the CBCT slices. All visible markers were detected by the navigation system, allowing automatic patient-to-image registration using point based rigid registration. The accuracy of the patient-to-image registration was evaluated and displayed during the experiment as the fiducial registration error $\left(\mathrm{FRE}_{\mathrm{NAV}}\right)$. Five trajectories, each defined by one target and one entry point, were then planned on CBCT slices using axial view. Finally, five series of five punctures each were performed on the phantom, which was fixed to a carbon plate together with the aiming device and placed on the CBCT table. At the completion of each puncture series, control MDCT scans were acquired for evaluation of positioning accuracy.

\section{Evaluation}

All CBCT-guided positioning errors were evaluated on control CT scans and compared to previous study results presented in Wallach et al. [16]. Expected positioning error pertaining to fused CBCT-CT guidance was deduced from an evaluation of positioning errors calculated on coregistered control CT and preoperative CBCT images.

Herein, ${ }^{\text {source }} p=(x, y, z)^{T} \in R^{3}$ denotes a point in the source coordinate system and ${ }^{\text {target }} T_{\text {source }}$ denotes the transformation matrix relating source to target coordinate system. In contrast, all scalars are denoted as non-bold letters with uppercase subscripts.

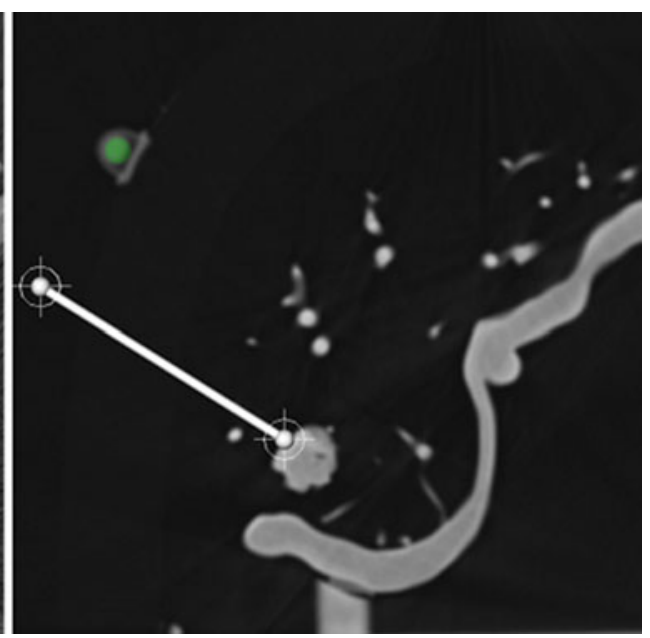

images. However they have 6-times higher longitudinal resolution and nearly 1.5-times higher in-plane resolution 
CBCT guidance positioning accuracy

The target positioning error $\left(\mathrm{TPE}_{\mathrm{CBCT}}\right)$, as described in Widmann et al. [21], was measured as the distance (vector norm, $\|\cdot\|$ ) between the final needle tip position ${ }^{\mathrm{ct}} p_{(\mathrm{nt})}$ and the target position ${ }^{\mathrm{ct}} p_{(\mathrm{pt})}$ on the control CT scan using a custom software tool, as per Eq. 1.

$\mathrm{TPE}_{\mathrm{CBCT}}=\left\|{ }^{\mathrm{ct}} p_{(\mathrm{pt})}-{ }^{\mathrm{ct}} p_{(\mathrm{nt})}\right\|$

$\mathrm{TPE}_{\mathrm{CBCT}}$ was separated into longitudinal (along the planned trajectory) and lateral components (along the orthogonal direction).

\section{CBCT-CT guidance positioning accuracy}

For the deduction of CBCT-CT guidance accuracy, CBCT volumes used for planning were registered to each of the five control CT scans. The rigid transformations ( $\left.{ }^{\mathrm{cbct}} T_{\mathrm{ct}}\right)$ mapping any point from ct to cbct coordinate system (Eq. 2) were calculated by pair point rigid matching of the SM positions located in the CT images $\left({ }^{\mathrm{ct}} p_{(\mathrm{sm})}\right)$ to the corresponding five SM positions in the CBCT images ( $\left.{ }^{\text {cbct }} p_{(\mathrm{sm})}\right)$. ${ }^{\mathrm{cbct}} p_{(\mathrm{sm})}={ }^{\mathrm{cbct}} T_{\mathrm{ct}} \cdot{ }^{\mathrm{ct}} p_{(\mathrm{sm})}$

Using the determined transformations, TPE $\mathrm{CBCT-СТ}_{\text {was }}$ calculated as the distance between the transformed needle tip from the CT scan and the planned target positions on the CBCT scan (refer to Fig. 4) as per Eq. 3

$\mathrm{TPE}_{\mathrm{CBCT}-\mathrm{CT}}=\left\|{ }^{\mathrm{cbct}} p_{(\mathrm{pt})}-{ }^{\mathrm{cbct}} T_{\mathrm{ct}} \cdot{ }^{\mathrm{ct}} p_{(\mathrm{nt})}\right\|$

where ${ }^{\mathrm{ct}} p_{(\mathrm{nt})}$ is the obtained needle tip position measured in the CT image, ${ }^{\text {cbct }} p_{(\mathrm{pt})}$ is the planned target position defined in the CBCT image, ${ }^{\text {cbct }} T_{\text {ct }}$ is an affine transformation matrix determined from rigid image co-registration, and the vector norm is denoted as $\|\cdot\|$.

The quality of the registration was evaluated through the fiducial image coregistration error $\left(\mathrm{FRE}_{\mathrm{CBCT}-\mathrm{CT}}\right)$, which was computed as the RMS error between registered corresponding landmarks. Furthermore the contribution of registration error to the overall positioning error was determined via computation of the target registration error $\left(\mathrm{TRE}_{\mathrm{CBCT-CT}}\right)$ for each of the five metal screws as the distance between registered corresponding targets as per Eq. 4

$\mathrm{TRE}_{\mathrm{CBCT}-\mathrm{CT}}=\left\|{ }^{\mathrm{cbct}} p_{(\mathrm{t})}-{ }^{\mathrm{cbct}} T_{\mathrm{ct}} \cdot{ }^{\mathrm{ct}} p_{(\mathrm{t})}\right\|$

where ${ }^{\text {ct }} p_{(\mathrm{t})}$ is the target position on CT images and ${ }^{\text {cbct }} p_{(\mathrm{t})}$ is the target position on CBCT images.

Statistical analysis

All $\mathrm{TPE}_{\mathrm{CBCT}}$ and $\mathrm{TPE}_{\mathrm{CBCT}-\mathrm{CT}}$ errors were described quantitatively using mean $(\mu)$ and standard deviation $(\sigma)$ as well as a maximum error value. Statistically significant differences were tested with the two-tailed, nonparametric, unpaired $t$ test, in which $p<0.05$ was defined as statistically significant.

\section{Results}

Despite the limited FOV of the CBCT device, five of the six SM spheres placed on the phantom surface could still be visualized within the resulting dataset. The numerous streak artifacts introduced from metal screws on the phantom did not remarkably reduce the quality of images obtained with the CBCT modality and detection of the SM,

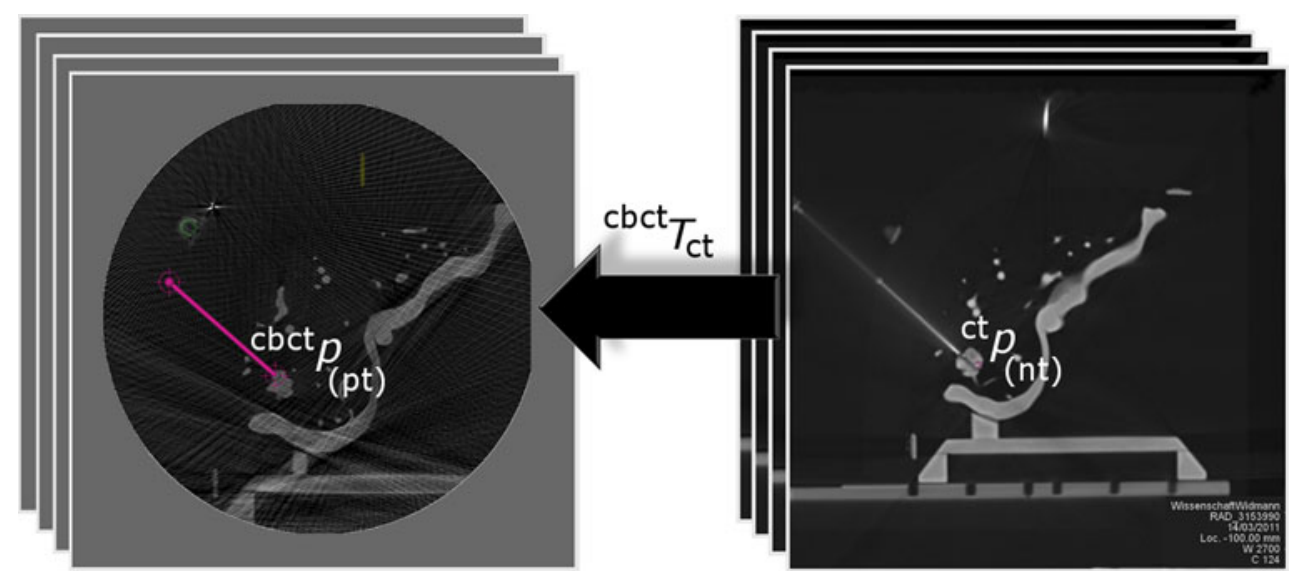

Fig. 4 CBCT-to-CT rigid image coregistration process for TPE evaluation. When SM-based image coregistration is performed, the achieved needle tip position ${ }^{c t} p_{(\mathrm{nt})}$ in a ct coordinate system (right) is transformed via the affine transformation matrix ${ }^{\mathrm{cbct}} T_{\mathrm{ct}}$ into the cbct coordinate system (left) and compared with the planned target position represented by ${ }^{\text {cbct }} p_{(\mathrm{pt})}$ 


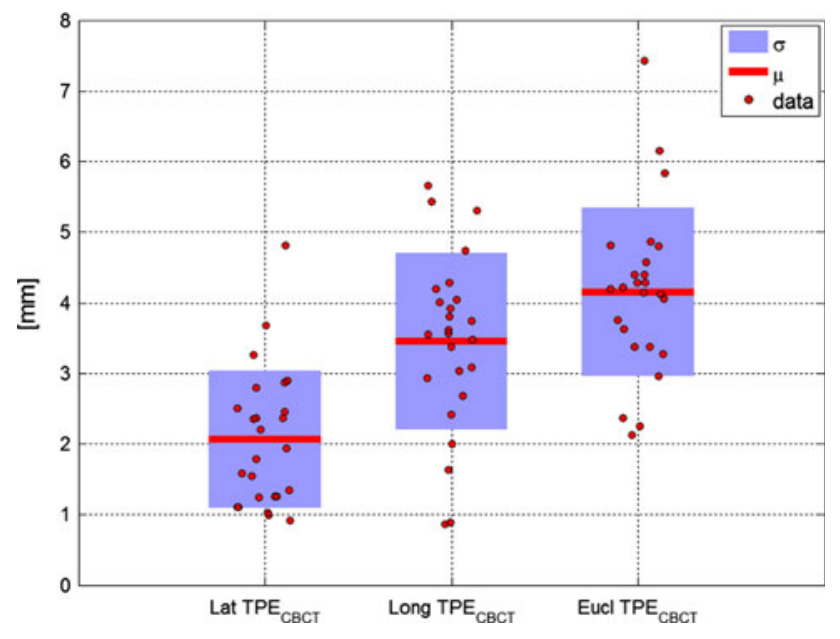

Fig. 5 Statistical analysis (mean $\mu$ and standard deviation $\sigma$ ) along different directions (lateral, longitudinal, and Euclidean) of ТРЕ

as well as the definition of targets and trajectories for navigated needle punctures, was possible. The precision of the target center selection on the CBCT slices was higher than on MDCT slices due to the finer spatial and longitudinal resolution of the former $(0.48 \times 0.48 \times 0.48 \mathrm{~mm})$ compared with the latter $(0.65 \times 0.65 \times 3 \mathrm{~mm}$; Fig. 3$)$.

The average patient to image registration matching error $\left(\mathrm{FRE}_{\mathrm{NAV}}\right)$ for all five series measured immediately before needle insertion was $0.5 \pm 0.1 \mathrm{~mm}$, with a maximum value of $0.9 \mathrm{~mm}$. The Euclidean distance between the achieved needle tip and target position measured on the CBCT images $\left(\mathrm{TPE}_{\mathrm{CBCT}}\right.$ ) was $4.1 \pm 1.0 \mathrm{~mm}$ (maximum $7.3 \mathrm{~mm}$ ), with lateral $\mathrm{TPE}_{\mathrm{CBCT}}$ of $2.1 \pm 1.0 \mathrm{~mm}$ (maximum $4.7 \mathrm{~mm}$ ) and longitudinal $\mathrm{TPE}_{\text {СвСт }}$ of $3.3 \pm 1.2 \mathrm{~mm}$ (maximum $5.7 \mathrm{~mm}$ ). All TPE $\mathrm{CBCT}_{\text {are presented in Fig. } 5 .}$

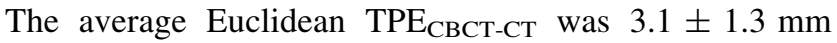
(maximum $6.2 \mathrm{~mm}$ ), with a lateral ТРЕ $_{\text {СвСт-Ст of }}$ $2.1 \pm 0.8 \mathrm{~mm}$ (maximum $4.0 \mathrm{~mm}$ ) and longitudinal $\mathrm{TPE}_{\text {СвСТ-Ст }}$ of $2.3 \pm 1.3 \mathrm{~mm}$ (maximum $5.4 \mathrm{~mm}$ ).

The average image coregistration error $\left(\mathrm{FRE}_{\mathrm{CBCT}-\mathrm{CT}}\right)$ was $0.3 \pm 0.1 \mathrm{~mm}$ (maximum value of $0.4 \mathrm{~mm}$ ). The TRE $_{\text {Свст-С }}$ computed at the five targets was $2.1 \pm 0.7 \mathrm{~mm}$ (maximum value of $3.6 \mathrm{~mm}$ ). Figure 6 presents the statistical distribution of all acquired TPE $_{\text {СвCT-СT }}$ errors and their comparison to TRE ${ }_{\text {СBCT-СT. }}$. Positioning accuracies pertaining to CBCT and CBCT-CT were found to be better or equivalent to those achieved with CT in both the lateral and longitudinal directions. The

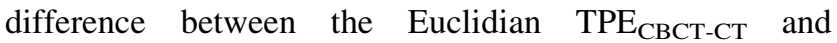
$\mathrm{TPE}_{\mathrm{CBCT}}$ errors was found to be statistically significant $(p=0.0037)$. The lateral components, however, were not statically significantly different $(p=1)$. A summary of all results is presented in Table 1.

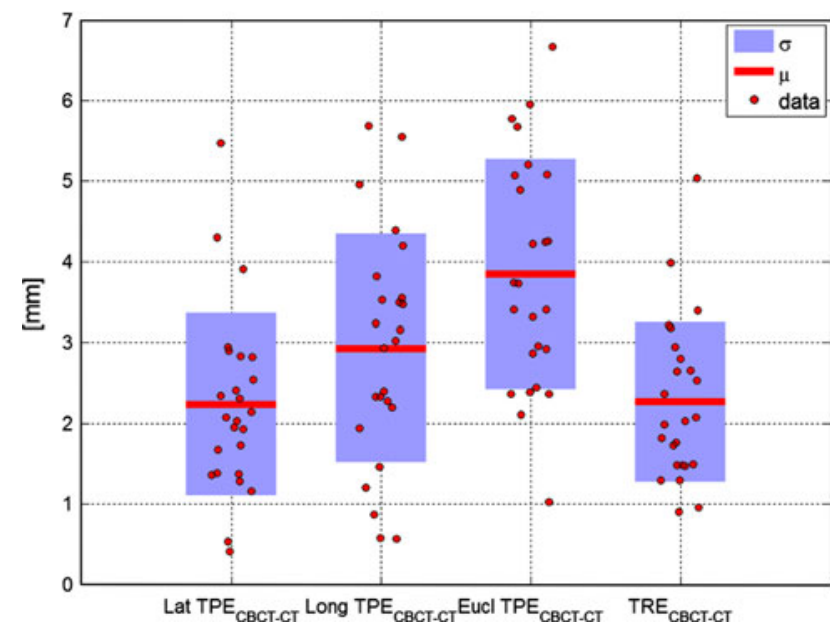

Fig. 6 Statistical analysis (mean $\mu$ and standard deviation $\sigma$ ) along different directions (lateral, longitudinal, and Euclidean) of $\mathrm{TPE}_{\mathrm{CBCT}-\mathrm{CT}}$ and TRE $\mathrm{CBCT}_{\text {-CT }}$

Table 1 Summary of all positioning accuracies from this study and a previously performed study [16]

\begin{tabular}{llll}
\hline & $\begin{array}{l}\mathrm{TPE}_{\mathrm{CT}}[16] \\
(\mathrm{mm})\end{array}$ & $\begin{array}{l}\mathrm{TPE}_{\mathrm{CBCT}} \\
(\mathrm{mm})\end{array}$ & $\begin{array}{l}\mathrm{TPE}_{\mathrm{CBCT}-\mathrm{CT}} \\
(\mathrm{mm})\end{array}$ \\
\hline Lateral & $2.3 \pm 1.3^{*}$ & $2.1 \pm 1.0^{*}$ & $2.1 \pm 0.8$ \\
Longitudinal & $3.7 \pm 1.3$ & $3.3 \pm 1.2$ & $2.3 \pm 1.3$ \\
Euclidean & $4.6 \pm 1.2$ & $4.1 \pm 1.0$ & $3.1 \pm 1.3$
\end{tabular}

* Difference between lateral targeting accuracies of CT-guided and CBCT-guided needle punctures are not statistically significant $(p=0.5155)$

\section{Discussion}

The clinical outputs of minimally invasive percutaneous thermal ablation of hepatic malignancies are highly dependent on applicator targeting accuracy. To enable accurate placement of the ablation needles, the use of imaging modalities such as contrast-enhanced MDCT might be of interest [9]. However, the use of CT scanners for radiological interventions is time-consuming and requires extensive human resources. This might lead to conflicts in schedules and restricts the availability of CT scanners in most clinical facilities.

The newest developments in C-arm CBCT that employ FPDs for large field-of-view volumetric imaging could possibly overcome these limitations [12]. Current systems for radiological interventions based on $\mathrm{C}$-arm $\mathrm{CT}$ devices provide 3D imaging capabilities combined with real-time 2D orthogonal fluoroscopy images to facilitate needle placement $[3,22]$. Placing the FPD orthogonally to the needle trajectory, needle adjustment and advancement can be overseen from entry point of view ("bull's eye view") or view perpendicular to the entry view, along the needle 
trajectory ("progression view"). Such a treatment modality, using the XperGuide system (Allura Xper FD20, Philips, The Netherlands), was successfully evaluated on 12 patients, stating a mean targeting accuracy of $2.8 \pm 2.4 \mathrm{~mm}$ (range, 0.5-9.4 mm) for soft tissue targets [14]. In a study with 139 patients, Braak et al. [13] reported a success of $100 \%$ in reaching the planned targets, using the previously mentioned 3D fluoroscopy-based needle guidance. However, a detailed evaluation of targeting accuracy was not presented. The main drawback of real-time fluoroscopy-guided procedures is the high patient and medical team overdosage during manual trajectory alignment and needle progression. Although it has been reported that patient irradiation during abdominal CBCT is comparable to that for MDCT, a computation of radiation dose to which the patient and medical staff are exposed during radiological interventions is very complex [2]. In contrast, use of an optical navigation system with stereotactic aiming device allows for trajectory alignment and needle stabilization at the highest possible accuracy without need for fluoroscopy guidance.

In the present study, average CBCT guidance accuracy $\left(\mathrm{TPE}_{\mathrm{CBCT}}\right)$ was evaluated on $\mathrm{CT}$ images. Therefore, the study outcomes (mean $4.1 \pm 1.0 \mathrm{~mm}$, maximum $7.3 \mathrm{~mm}$ ) could be compared to results previously reported in literature using CT-based navigation [16, 23, 24]. Maier-Hein et al. [23] reported an average TPE of $3.5 \pm 1.1 \mathrm{~mm}$ from 20 in vitro needle insertions in three porcine livers containing tumor-mimicking agar nodules. Livers were mounted to the artificial diaphragm placed inside the anthropomorphic respiratory motion simulator. In a secondary in vivo experiment, in which 32 freehand needle punctures were performed on ventilated swine, overall errors of $3.7 \pm 2.3 \mathrm{~mm}$ (maximum error of $11.06 \mathrm{~mm}$ ) were reported [24]. For both studies, neither lateral nor longitudinal components were computed. Dividing TPE into individual longitudinal and lateral components is clinically relevant due the fact that, in contrast to the correction of longitudinal placement errors (depth of the needle), lateral errors requires repositioning of the needle, which could be a potential source of risk and complication.

Additionally, we have previously reported lateral $\mathrm{TPE}_{\mathrm{CT}}$ of $2.3 \pm 1.3 \mathrm{~mm}$ evaluated on CT control scans [16] versus the $2.1 \pm 1.0 \mathrm{~mm}$ for the lateral $\mathrm{TPE}_{\mathrm{CBCT}}$ measured with CBCT guidance in the presented study (Table 1). A two-tailed $t$ test showed that the difference between both sets of results was not statistically significant ( $p=0.5155)$.

Concerning imaging protocols, CBCT devices still do not provide as good contrast-to-noise ratio or artifact level as MDCT scanners. Motion artifacts also are much more prominent with $\mathrm{C}$-arm devices while sensitivity of FPDs is much lower than those used in multidetector row scanners as has been shown from preliminary experience in abdominal interventional procedures [25]. It may therefore be necessary to fuse intraoperative CBCT data with preoperative MDCT scan data for better tumor visualization, accurate treatment planning, as well as final ablation zone control. On the other hand, CB-CT has not been shown to be negatively affected by thermal ablation within preliminary experience in abdominal interventional procedures [26].

Rigid, landmark-based, image coregistration methods have shown an acceptable accuracy for fusion of diagnostic and postoperative CT scans. In Fujioka et al. [27], the liver border and vessels close to the tumor have been used as landmarks. The maximal gap recorded between segmented organs was within the range of $1-5 \mathrm{~mm}$, considering that there was no motion control during acquisition. In order to diminish the effects of organ motion, deformable (nonrigid) CBCT-CT image coregistration methods have been developed and successfully applied to adaptive radiotherapies providing a tool for dose summation throughout the entire therapy sessions [28, 29]. Deformable CBCT-CT coregistration methods also have been used for automatic target delineation in head-and-neck cancer [30], yielding a TRE of $2.8 \pm 0.2 \mathrm{~mm}$ in soft tissue targets.

In the presented nonrigid liver phantom study, a fiducial marker-based registration was used. Unlike anatomical landmarks in which precise definition may be difficult, time-consuming and dependent on the experience of the operator the center of the fiducial markers can be easily defined in the image in minimal time [31]. Using the described methodology, an image coregistration error $\left(\right.$ FRE $\left._{\text {СBCT-CT }}\right)$ of $0.3 \pm 0.1 \mathrm{~mm}$ was achieved with corresponding TRE $\mathrm{E}_{\text {СвСт-Ст }}$ results for the presented fiducial and target configurations of $2.1 \pm 0.7 \mathrm{~mm}$. The experimentally determined TRE is higher than expected on a phantom due to the difficulty in obtaining a precise definition of the $1.5-\mathrm{mm}$ target screws using the 3-mm CT slices compared with the 0.48-mm resolution in the CBCT slices.

It should be noted that the measured $\mathrm{TRE}_{\mathrm{CBCT}-\mathrm{CT}}$ values do not include errors introduced by patient motion. The use of rigid image coregistration methods on a phantom with static targets does not clarify what the endpoint registration error will be when fusing CBCT of the soft tissue with diagnostic CT images. In case of large organ movements caused by patient transportation or difference in a patient position on a diagnostic CT (supine position) or in radiological suite (left-lateral recumbent, side position), such a coregistration method might be insufficient. Additionally, motion also could be related to long acquisition times and low detector sensitivity present in C-arm CBCT modality [12]. Therefore, appropriate respiratory gating during both acquisition protocols and patient stabilization via vacuum cushions during intervention is recommended [32].

Unexpectedly, the $\mathrm{TPE}_{\mathrm{CBCT-Cт}}$ pertaining to fused images was better or equivalent to those pertaining to CBCT alone $\left(\mathrm{TPE}_{\mathrm{CBCT}}\right)$. This may be due to the positioning error 
being partially compensated by image coregistration errors. This effect is particularly evident in the longitudinal direction in which expected registration error is higher due to a lower longitudinal resolution of the CT compared to CBCT.

Navigation systems help radiologists with pre- and intraoperative information interpretation, treatment planning and guidance of needles based on up-to-date image acquisitions. Compared with previous navigation systems that were adapted to the needs of interventional radiology, the presented navigation system is specifically designed for liver interventions. It is markedly reduced in size and implements dedicated software for tumor ablations. Furthermore, it allows for automated image-to-patient registration based on the SM, thus, a manual localization of registration markers with a tracked probe is no longer required.

The primary weakness of the presented phantom study is a lack of a realistic clinical scenario, which could provide information pertaining to the sufficiency of the quality of CBCT datasets from an angiography system for visualization of the liver lesions. However, usage of contrast solutions might be helpful to identify hypervascular tumors and accurately correlate their position to vascular anatomy $[15,26]$. Furthermore, we could not determine if the small FOV and limited detector range is sufficient to obtain proper data for needle guidance in clinical routine. It is required that both SM spheres on the patient skin and targeting tumors need to be visible on the acquired scan. Because of this, an exact positioning of the patient on the angiography table is required, which may be quite timeconsuming or even impossible for obese patients. Secondly, it is not yet clear from a clinical point of view whether placing the SM on the patient skin for the diagnostic CT, which is typically acquired a few days before the intervention, will be feasible due to unattended SM displacement and possible patient discomfort. Therefore, implementation of marker-free registration methods is being investigated as ongoing research.

\begin{abstract}
Acknowledgments Parts of the work presented in this manuscript are founded by the Eurostars-Eureka Programme under the project iVisc (E!6201). The authors thank CAScination AG, Bern, Switzerland, for providing hardware support, Dr. Matthias Peterhans and Sylvain Anderegg for valuable support during the development of the approach, and finally Kate A. Gavaghan and Tom Williamson for proofreading the paper.
\end{abstract}

Conflict of interest Grzegorz Toporek, Dr. Daphné Wallach, Prof. Dr. Stefan Weber, and Dr. Gerlig Widmann declare no conflict of interest. Prof. Reto Bale is a coinventor of the stereotactic ATLAS aiming device (Medical Intelligence $\mathrm{GmbH}$, Schwabmünchen, Germany) and a coshareholder in its financial returns.

\section{References}

1. McCarley JR, Soulen MC (2010) Percutaneous ablation of hepatic tumors. Sem Interv Radiol 27:255-260
2. Racadio JM, Babic D, Homan R, Rampton JW, Patel MN, Racadio JM, Johnson ND (2007) Live 3D guidance in the interventional radiology suite. AJR Am J Roentgenol 189:W357W364

3. Wood BJ, Kruecker J, Locklin J, Levy E, Xu S, Solbiati L, Amalou H, Venkatesan A (2011) Navigation systems for ablation. J Vasc Interv Radiol 21:1-19

4. Minami Y, Kudo M, Kawasaki T, Chung H, Ogawa C, Shiozaki H (2004) Percutaneous radiofrequency ablation guided by contrast-enhanced harmonic sonography with artificial pleural effusion for hepatocellular carcinoma in the hepatic dome. AJR Am J Roentgenol 182:1224-1226

5. Dill-Macky MJ, Asch M, Burns P, Wilson S (2006) Radiofrequency ablation of hepatocellular carcinoma: predicting success using contrast-enhanced sonography. AJR Am J Roentgenol 186(5 Suppl):S287-S295

6. van den Bosch M, Daniel B, Rieke V, Butts-Pauly K, Kermit E, Jeffrey S (2008) MRI-guided radiofrequency ablation of breast cancer: preliminary clinical experience. J Magn Reson Imaging 27:204-208

7. Livraghi T, Solbiati L, Meloni F, Ierace T, Goldberg SN, Gazelle GS (2003) Percutaneous radiofrequency ablation of liver metastases in potential candidates for resection: the "test-of-time approach". Cancer 97:3027-3035

8. Dromain C, De Baere T, Elias D, Kuoch V, Ducreux M, Boige V, Petrow P, Roche A, Robert S (2002) Hepatic tumors treated with ablation: CT and MR imaging. Radiology 223:255-262

9. Kim SK, Lim HK, Kim YH, Lee WJ, Lee SJ, Kim SH, Lim JH, Kim SA (2003) Hepatocellular carcinoma treated with radiofrequency ablation: spectrum of imaging findings. Radiographics 23:107-121

10. Oliveira-Santos T, Klaeser B, Weitzel T, Krause T, Nolte LP, Peterhans M, Weber S (2011) A navigation system for percutaneous needle interventions based on PET/CT images: design, workflow and error analysis of soft tissue and bone punctures. Comput Aided Surg 16:203-219

11. Meier-Meitinger M, Nagel M, Kalender W, Bautz WA, Baum U (2008) Computer-assisted navigation system for interventional CT-guided procedures: results of phantom and clinical studies. RöFo 180:310-317

12. Orth RC, Wallace MJ, Kuo MD (2008) C-arm cone-beam CT: general principles and technical considerations for use in interventional radiology. J Vasc Interv Radiol 19:814-820

13. Braak SJ, van Strijen MJL, van Leersum M, van Es HW, van Heesewijk JPM (2010) Real-time 3D fluoroscopy guidance during needle interventions: technique, accuracy, and feasibility. AJR Am J Roentgenol 194:445-451

14. Leschka SC, Babic D, El Shikh S, Wossmann C, Schumacher M, Taschner C (2012) C-arm cone beam computed tomography needle path overlay for image-guided procedures of the spine and pelvis. Neuroradiology 54:215-223

15. Wallace MJ, Kuo MD, Glaiberman C, Binkert CA, Orth RC, Soulez G (2008) Three-dimensional C-arm cone-beam CT: applications in the interventional suite. J Vasc Interv Radiol 19:799-813

16. Wallach D, Toporek G, Weber S, Bale R, Widmann G (2013) Comparison of free-hand navigated and aiming device navigated targeting of liver lesions. Int J Med Robot Comput Assist Surg (in press)

17. Peterhans M, vom Berg A, Dagon B, Inderbitzin D, Baur C, Candinas D, Weber S (2011) A navigation system for open liver surgery: design, workflow and first clinical applications. Int J Med Robot Comput Assist Surg 7(1):7-16

18. Tschannen M, Toporek G, Wallach D, Peterhans M, Weber S (2012) Single-marker localization for automatic patient registration in interventional radiology. In: CURAC 2012, Düsseldorf 
19. Oliveira-Santos T, Peterhans M, Hofmann S, Weber S (2011) Passive single marker tracking for organ motion and deformation detection in open liver surgery. In: Proceedings of the second international conference on information processing in computerassisted interventions, pp 156-167

20. Widmann G, Schullian P, Ortler M, Bale R (2012) Frameless stereotactic targeting devices: technical features, targeting errors and clinical results. Int J Med Robot Comput Assist Surg 8:1-16

21. Widmann G, Stoffner R, Sieb M, Bale R (2009) Target registration and target positioning errors in computer-assisted neurosurgery: proposal for a standardized reporting of error assessment. Int J Med Robot Comput Assist Surg 5:355-365

22. Möhlenbruch M, Nelles M, Thomas D, Willinek W, Gerstner A, Schild HH, Wilhelm K (2010) Cone-beam computed tomography-guided percutaneous radiologic gastrostomy. Cardiovasc Interv Radiol 33:315-320

23. Maier-Hein L, Pianka F, Seitel A, Müller SA, Tekbas A, Seitel M, Wolf I, Schmied BM, Meinzer H (2007) Precision targeting of liver lesions with a needle-based soft tissue navigation system. Med Image Comput Comput Assist Interv 10(Pt 2):42-49

24. Maier-Hein L, Tekbas A, Seitel A, Pianka F, Müller SA, Satzl S, Schawo S, Radeleff B, Tetzlaff R, Franz AM, Müller-Stich BP, Wolf I, Kauczor HU, Schmied BM, Meinzer HP (2008) In vivo accuracy assessment of a needle-based navigation system for CT-guided radiofrequency ablation of the liver. Med Phys 35:5385-5396

25. Morimoto M, Numata K, Kondo M, Nozaki A, Hamaguchi S, Takebayashi S, Tanaka K (2010) C-arm cone beam CT for hepatic tumor ablation under real-time 3D imaging. AJR Am J Roentgenol 194:W452-W454

26. Hirota S, Nakao N, Yamamoto S, Kobayashi K, Maeda H, Ishikura R, Miura K, Sakamoto K, Ueda K, Baba R (2006) Conebeam $\mathrm{CT}$ with flat-panel-detector digital angiography system: early experience in abdominal interventional procedures. Cardiovasc Interv Radiol 29:1034-1038

27. Fujioka C, Horiguchi J, Ishifuro M, Kakizawa H, Kiguchi M, Matsuura N, Hieda M, Tachikake T, Alam F, Furukawa T, Ito K (2006) A feasibility study: evaluation of radiofrequency ablation therapy to hepatocellular carcinoma using image registration of preoperative and postoperative CT. Acad Radiol 13:986-994

28. Lawson JD, Schreibmann E, Jani AB, Fox T (2007) Quantitative evaluation of a cone-beam computed tomography-planning computed tomography deformable image registration method for adaptive radiation therapy. J Appl Clin Med Phys 8:2432

29. Paquin D, Levy D, Xing L (2009) Multiscale registration of planning CT and daily cone beam CT images for adaptive radiation therapy. Med Phys 36:4

30. Hou J, Guerrero M, Chen W, D'Souza WD (2011) Deformable planning CT to cone-beam CT image registration in head-andneck cancer. Med Phys 38:2088-2094

31. Kral F, Url C, Widmann G, Riechelmann H, Freysinger W (2011) The learning curve of registration in navigated skull base surgery. Laryngorhinootologie 90:90-93

32. Widmann G, Schullian P, Haidu M, Wiedermann FJ, Bale R (2010) Respiratory motion control for stereotactic and robotic liver interventions. Int J Med Robot 6:343-349 